\title{
Increasing fungal abundance in the substrate enhances seed germination in a fully mycoheterotrophic orchid
}

\author{
Yuan-Yuan $\mathrm{Li}^{1}$, Margaux Boeraeve ${ }^{2}$, Yu-Hsiu Cho ${ }^{3}$, Hans Jacquemyn ${ }^{4}$, and Yung-I Lee ${ }^{3}$ \\ ${ }^{1}$ China Agricultural University College of Plant Protection \\ ${ }^{2}$ Katholieke Universiteit Leuven Faculteit Wetenschappen \\ ${ }^{3}$ National Museum of Natural Science \\ ${ }^{4}$ University of Leuven
}

May 17, 2021

\begin{abstract}
Mycorrhizal associations are essential for orchid germination and seedling establishment, and thus may constrain the distribution and abundance of orchids under natural conditions. Previous works have shown that germination and seedling establishment in several orchids often decline with increasing distance from adult plants, resulting in nonrandom spatial patterns of seedling establishment. In contrast, individuals of the fully mycoheterotrophic orchid Gastrodia confusoides often tend to have random spatial patterns of distribution within bamboo forests. Since G. confusoides is parasitic on litter-decaying fungi, its random spatial patterns of distribution may be due to highly scattered patterns of litter-decaying fungi within bamboo forests. To test this hypothesis, we first identified the main mycorrhizal fungi associating with developing seeds and adult plants using Miseq high-throughput sequencing. Next, we combined seed germination experiments with quantitative PCR (qPCR) analyses to investigate to what extent the abundance of mycorrhizal fungi affected spatial patterns of seed germination. Our results show that seed germination and subsequent growth to an adult stage in G. confusoides required a distinct switch in mycorrhizal partners, in which protocorms associated with a single Mycena fungus, while adults associated with a fungus from the genus Gymnopus. A strong, positive relationship was observed between germination and Mycena abundance in the litter, but not between germination and Gymnopus abundance. Fungal abundance was not significantly related to the distance from the adult plants, and consequently germination was also not significantly related to the distance from adult plants. Our results provide the first evidence that the spatial distribution and abundance of litter-decaying fungi are distributed randomly within the bamboo forest and independently from G. confusoides adults.
\end{abstract}

\section{INTRODUCTION}

Both dispersal and recruitment limitation represent major factors that affect the distribution and abundance of plant populations (Eriksson \& Ehrlén, 1992; Clark et al., 2007). Dispersal of seeds into favorable microsites is a first critical step shaping recruitment and distribution patterns of plants (Conditet al., 1992; Nathan \& Muller-Landau, 2000; Wang \& Smith, 2002; Getzin et al., 2014). When seeds fail to reach suitable habitats, no recruitment will occur at these sites and plants are considered dispersal or seed limited (Clark et al.,2007; Poulsen et al., 2007). If, on the other hand, seeds are widely dispersed, but fail to recruit because habitats are unsuitable, plants are considered habitat- or microsite-limited (Münzbergová \& Herben, 2005; Johansson \& Eriksson, 2013). Most seed introduction and addition experiments have shown that supplemental addition of seeds to populations almost always results in increased seedling recruitment, indicating that the majority of plant populations are to some extent seed limited (Clark et al., 2007; Poulsen et al., 2007).

For plant species that critically depend on mycorrhizal symbiosis for seed germination and seedling establishment, the availability of appropriate mycorrhizal fungi may represent an influential portion of favorable 
microsite, and this is particularly true for orchids (Diez, 2007; Jacquemyn et al., 2007; McCormick \& Jacquemyn, 2014; Waud et al., 2016; McCormick et al., 2018). Orchids have dust-like seeds that consist of rudimentary embryos without endosperm at the time of seed dispersal (Arditti \& Ghani, 2000). Under natural conditions, the germination of orchid seeds and the early development of protocorms rely on mycorrhizal associations for nutrient supplies (Smith \& Read, 2008; Rasmussen \& Rasmussen, 2009; Rasmussen et al., 2015). Therefore, the availability of suitable mycorrhizal fungi is considered to be a limiting factor in seedling recruitment that significantly affects the abundance and spatial distribution of orchids (Rasmussen \& Whigham, 1998; McKendrick et al., 2002; McCormick \& Jacquemyn, 2014; McCormick et al., 2018).

Because orchid mycorrhizal (OrM) fungi can grow well without the association with orchids, it is generally assumed that OrM fungi are distributed independently from orchids and are therefore more widespread than the distribution of orchids suggests (McCormick et al., 2018). Previous studies using seed introduction experiments have indeed shown that the distribution of orchids was rarely limited by the availability of suitable OrM fungi at a landscape scale (McCormick \& Jacquemyn, 2014). At the local scale, however, seed germination is often erratic, suggesting that OrM fungi are not regularly distributed within sites. Results from previous studies have, for example, shown that germination and seedling establishment in photosynthetic orchids often decline with increasing distance from adult orchids (Batty et al., 2001; Diez, 2007; Jacquemyn et al., 2007, 2012a,b; McCormick et al., 2012). Because most green adult orchids maintain associations with mycorrhizal fungi and in this way possibly establish a mutualistic symbiosis with their rhizoctonia fungal symbionts, this may create favorable microsites for seed germination near the rhizosphere of adult orchids (Cameron et al., 2008; Smith \& Read, 2008; McCormick et al., 2016; Waud et al., 2016).

Mixed results have been obtained for fully mycoheterotrophic orchids, which are species that are nonphotosynthetic throughout their entire life and solely depend on fungal carbon. In Neottia nidus-avis , for example, significant differences in seed germination and seedling development were observed between plots containing adult $N$. nidus-avis plants and plots situated at least $5 \mathrm{~m}$ from the nearest adult, but there was no significant difference in seed germination between seed packets buried within $15 \mathrm{~cm}$ of the adults and packets buried at 15-25 cm from adult plants (McKendrick et al., 2002). In Coralorrhiza trifida, on the other hand, there was no significant relationship between the extent of germination or the rate of development and the presence of naturally distributed plants at a spatial scale of $1 \mathrm{~m}$ (McKendrick et al., 2000), suggesting that growth of $C$. trifida seedlings depends more on the ability of the fungal symbionts to transfer carbon from their ectomycorrhizal co-associates than on the distribution of conspecific adult plants.

In this study, we investigated the abundance and distribution of mycorrhizal fungi in bamboo litter and how they relate to spatial patterns of seed germination of the fully mycoheterotrophic orchid Gastrodia confusoides . A number of species of the genus Gastrodia exclusively inhabit bamboo forests and are parasitic on litterdecaying fungi to gain access to nutrients through the degradation of bamboo leaf and twig litter, while the litter-decaying fungi are most likely not dependent on this association for survival (Kinoshita et al., 2016). Given that the ground surface of bamboo forests is densely covered by bamboo leaf and twig litter, and litter-decaying fungi may show highly scattered patterns within the bamboo forest, we hypothesized that germination is likely more dependent on fungal abundance in the litter than on the distance to the nearest adult plant. To test this hypothesis, we used high-throughput sequencing to compare the fungal communities associating with protocorms and adults to identify the mycorrhizal fungi promoting seed germination in $G$. confusoides. We further combined seed germination experiments with quantitative PCR (qPCR) analyses to investigate spatial variation in fungal abundance within the surrounding bamboo litter at different distances from adult plants and to assess how spatial variation in fungal abundance affected seed germination.

\section{MATERIAL AND METHODS}

\subsection{Study species}

The genus Gastrodia consists of about 66 species that are widely distributed across the Paleotropics, Oceania, and warm-temperate regions in Eastern Asia (Pridgeon et al., 2005; WCSP, 2014). All of them are all fully mycoheterotrophic plants that occur in moist and sheltered forests. Gastrodia species have been reported to 
associate with litter-decaying fungi and/or wood-decaying fungi (Kusano, 1911; Ogura-Tsujita et al., 2009; Martos et al., 2009; Dearnaley \& Bougoure, 2010; Lee et al., 2015). Interestingly, several Gastrodiaspecies, such as Gastrodia appendiculata, Gastrodia confusoides and Gastrodia nantoensis occur predominantly in bamboo forests in Taiwan (Hsu, 2008) and litter-decaying fungi of the fungal genera Mycena and Marasmia have been identified as their major mycorrhizal partners (Ogura-Tsujita et al., 2009; Lee et al., 2015).

Gastrodia confusoides is a fully mycoheterotrophic orchid that occurs mainly in bamboo forests in central Taiwan. The species generally flowers in September, producing short flowering stalks that vary in height between 2 and $5 \mathrm{~cm}$ (Fig. 1A). After successful pollination, the flowering stalks elongate rapidly and reach a height between 20 and $50 \mathrm{~cm}$ (Fig. 1B). Capsules ripen quickly after one month of pollination, followed by dehiscence and seed dispersal in October (Fig. 1C). Only flowers or elongated stalks with capsules of this fully mycoheterotrophic orchid can be found above-ground from September to October. After the seeds have been dispersed, successful development of underground tubers and roots depends on fungal associations with litter-decaying fungi (Fig. 1D, E).

\subsection{Seed germination experiment}

To investigate whether seed germination varied along the distance to the adult plant, a seed germination experiment was established in four plots using the seed package method described by Rasmussen \& Whigham (1993). Experiments were conducted in a bamboo forest located in Heping District, Taichung City, Taiwan at 1000 meter above sea level. The bamboo forest was dominated by the mōsō bamboo (Phyllostachys edulis ) on organic soil with only few understory plants. When seeds were mature at the beginning of October 2015, capsules were harvested from the studied populations. In order to exclude potential population effects that may create differences in seed quality, all seeds were pooled after harvesting and directly put into seed packages. Per seed package, approximately 500 seeds were placed within a square of $53-\mu \mathrm{m}$ mesh phytoplankton netting. Seed packages were buried into the litter layer in each cardinal direction (north, south, east, west) at five different distances $(5,50,100,200,500 \mathrm{~cm})$ around each sampled orchid adult of the same $10-\mathrm{m}^{2}$ plot where roots and litter samples were taken (Fig. S1). The adult orchids were selected randomly without visible neighboring adult orchids within a radius of $5 \mathrm{~m}$. In total, 5 (distances) $\mathrm{x} 4$ (directions) $\mathrm{x} 4$ (plots) $=80$ seed packages were left in the ground for about 1 year. In October 2016, seed packages were retrieved, gently washed and maintained moist in paper towel for 1 day until examination. In the laboratory, seed packages were gently rinsed with tap water, then opened and carefully checked under a stereo microscope for counting germination. As orchid seed germination stages can be variable, germination was considered to have occurred when clear signs of mycorrhiza formation were observed and the rupture of testa by enlarging embryos were present (Fig. 2).

\subsection{Sampling of mycorrhizal communities}

Litter samples were collected in each cardinal direction (north, south, east, west) at five different distances (5, $50,100,200,500 \mathrm{~cm}$ ) around each sampled orchid adult (Fig. S1). At each sampling point, litter samples (ca. $10 \mathrm{~g}$ fresh weight) were taken using a sterile sampling bag, and the littler samples were immediately stored at $-80{ }^{\circ} \mathrm{C}$ for subsequent analysis. Genomic DNA from litter samples was extracted using the PowerSoil ${ }^{\circledR}$ DNA Isolation Kit (MO BIO Laboratories Inc., Carlsbad, CA). Additionally, at least three roots from each sampled orchid adult plant were surface sterilized with a $1 \%$ sodium hypochlorite solution for $30 \mathrm{~s}$, followed by three 30-s rinse steps in sterile distilled water and microscopically checked for mycorrhizal colonization. Subsequently, the distal 5-cm portions of colonized roots were sectioned into 3-5 mm fragments, which were then pooled to create a homogeneous mycorrhizal root sample for each individual plant. The protocorms from seed packets in each plot were pooled because of the small amount of tissue. The protocorms were rinsed with water to remove excess soil and litter fragments and were surface sterilized as root samples described above. Afterwards, DNA was extracted from $0.1 \mathrm{~g}$ mycorrhizal root fragments or protocorms using the Plant Genomic DNA Purification Kit as described by the manufacturer (GMbiolab Co. Ltd., Taichung, Taiwan). Altogether, there were 88 unique samples ( 4 adult root samples, 4 protocorm samples, 20 litter samples per G. confusoides plot) for DNA extraction and subsequent molecular analysis. 


\subsection{Molecular methods}

The ITS1 region of the nuclear ribosomal RNA genes was amplified using the primer pair ITS1F and ITS2R (Table S1; Adams et al., 2013). PCR was carried out in $20 \mu \mathrm{l}$ reactions containing $10 \mathrm{ng}$ of genomic DNA, 0.8 $\mu \mathrm{L}$ of each primer $(5 \mu \mathrm{M}), 2 \mu \mathrm{L}$ of $2.5 \mathrm{mM}$ dNTPs, $4 \mu \mathrm{L}$ of $5 \mathrm{x}$ TransStart ${ }^{\circledR}$ FastPfu Buffer (TransGen Biotech Co., Ltd., Beijing, China), and $0.4 \mu \mathrm{L}$ of TransStart ${ }^{\circledR}$ FastPfu Polymerase. The parameters of reactions consisted of an initial denaturation at $95{ }^{\circ} \mathrm{C}$ for $3 \mathrm{~min}$, then 35 cycles of $95{ }^{\circ} \mathrm{C}$ for $30 \mathrm{~s}$, and $55{ }^{\circ} \mathrm{C}$ for $30 \mathrm{~s}$ and a final extension of $72{ }^{\circ} \mathrm{C}$ for $45 \mathrm{~s}$. PCR products were separated by gel electrophoresis and amplicons within the appropriate size range were cut and purified using the AxyPrep DNA Gel Extraction Kit (Axygen Biosciences, Union City, CA) and quantified using QuantiFluor Fluorometer (Promega Corporation, Madison, WI). Samples were then pooled in equimolar concentrations and paired-end sequenced (2 x 250 bp) on an Illumina Miseq.

\subsection{Bioinformatics}

Raw fastq files were demultiplexed and quality-filtered using QIIME (version 1.17) with the following criteria. First, $300 \mathrm{bp}$ reads were truncated at any site receiving an average quality score $<20$ over a $50 \mathrm{bp}$ sliding window, discarding the truncated reads that were shorter than $50 \mathrm{bp}$. Second, reads with one or two nucleotide mismatches in primer matching measurements and reads containing ambiguous characters were removed. Third, only sequences that had an overlap longer than $10 \mathrm{bp}$ were assembled according to their overlap sequence. Reads that could not be assembled were discarded.

Sequences obtained from the Illumina Miseq run were clustered into operational taxonomic units (OTUs) using the UPARSE algorithm implemented in USEARCH version 7 (Edgar, 2013). OTUs were clustered with 97 $\%$ similarity cutoff using UPARSE (version $7.1 \mathrm{http}: / /$ drive5.com/uparse/) and chimeras were identified and removed using the UNITE UCHIME reference data set. The taxonomy of each ITS1 region was analyzed by RDP Classifier (http://rdp.cme.msu.edu/) against the UNITE fungal ITS database using confidence threshold of $70 \%$. Remaining OTUs were assigned taxonomic identities based on the top 10 BLAST (megablast algorithm) (Altschul et al., 1990) results of the OTU representative sequences (selected by UPARSE) using the GenBank nucleotide (nt) database (Benson et al., 2009), including uncultured/environmental entries. OTUs identified by BLAST were then manually screened for potential orchid-associating mycorrhizal families following a step-wise process. Firstly, OTUs represented by short sequences $(<150 \mathrm{bp})$ or having a low sequence similarity $(<90 \%)$ with fungal species across their sequence length were removed. Secondly, only OTUs which were found on at least one orchid root sample were retained. The results were put in an OTU table (sample x OTU table with each cell containing read numbers), which was used in the further analyses.

\subsection{Assessment of fungal abundance}

Illumina Miseq sequencing revealed that the most abundant OrM fungi associating with Gastrodia confusoides corresponded to two distinct saprotrophic non-rhizoctonia fungal taxa, i.e. Mycena(OTU3) and Gymnopus (OTU5). For both OTUs, a real-time quantitative PCR (qPCR) assay was developed and validated based on the internal transcribed spacer (ITS) 2 region (Table S1). The qPCR assays were utilized to quantify Mycena (OTU3) and Gymnopus(OTU5) DNA from each protocorm, root or litter DNA sample on an ABI QuantStudio 6 Flex real-time PCR instrument (Life Technologies, Carlsbad, CA, USA). Reactions were performed in a total volume of $25 \mu \mathrm{L}$ consisting of $12.5 \mu \mathrm{L}$ of iQ SYBR Green PCR Super Mix (Bio-Rad Laboratories, Hercules, CA, USA), $1.0 \mu \mathrm{L}$ DNA template, $9.0 \mu \mathrm{L} \mathrm{H}_{2} \mathrm{O}$ and $1.25 \mu \mathrm{L}(10 \mathrm{mM})$ of each forward and reverse primer, depending on the target OUT (Table S1). Amplifications were run as follows: initial denaturation for 5 min at $95{ }^{\circ} \mathrm{C}$, followed by 40 cycles of $15 \mathrm{~s}$ of denaturation at $94{ }^{\circ} \mathrm{C}, 30 \mathrm{~s}$ of annealing at 59 ${ }^{\circ} \mathrm{C}$ (OTU3 and OTU5), and $30 \mathrm{~s}$ of elongation at $72{ }^{\circ} \mathrm{C}$. Each sample extract was amplified in duplicate and quantified using a standard curve amplified in triplicate. Standard curves (range, 1.0E + $02-1.0 \mathrm{E}+06 \mathrm{~mol}$ $\mu 1^{-1}$ ) were generated using five 10-fold dilutions of target DNA amplified from protocorms (OTU3) or roots (OTU5). A melting curve analysis was performed after each analysis to confirm product specificity (Fig. S2A, C). In addition, amplification accuracy was verified by Sanger sequencing of a number of generated amplicons. In this way, product identity was confirmed forall samples considered to be positive using the 
qPCR assays (Ct value $<29$ and a single melting peak at the expected melting temperature; Fig. S2). Additional positive (DNA extract from protocorms colonized with OTU3 or roots colonized with OTU5) and negative control reactions (no template DNA) were included in triplicate with all analyses to verify target-specific amplification and the absence of contaminants, respectively.

\subsection{Data analysis}

To investigate spatial variation in mycorrhizal abundance and seed germination, we used Generalized Linear Mixed Models and Linear Mixed Models. First, a Linear Mixed Model was used to test the hypothesis that fungal abundance was related to the distance of the nearest adult plant. Fungal concentration $(\mathrm{mol} / \mu \mathrm{L})$ was used as dependent variables, while distance to adult plants was used a fixed factor. Plot was included in the model as a random factor. Analyses were performed separately for the main fungus associating with protocorms and the fungus associating with adult plants. A Generalized Linear Mixed Model was used to see whether seed germination (at least one seed developing into protocorm) was related to the distance of the nearest adult plant or to the abundance of key fungi (Mycena and Gymnopus) in the litter. Presence/absence of at least one protocorm in seed packages was used as dependent variable, while plot was included as a random effect in the model. In a second analysis, we related germination success (i.e., the percentage of seeds developing into a protocorm) to the distance of the nearest adult plant and the abundance of fungi in the soil.

\section{RESULTS}

\subsection{Mycorrhizal associations}

The quality-filtered Miseq data set contained samples from protocorms, adult roots and surrounding bamboo litter. The protocorm samples comprised 129 OTUs (172552 sequences), while the adult root samples comprised 427 OTUs (168843 sequences) (Table S2). The surrounding bamboo litter samples comprised 3925341 sequences with 1640 OTUs (excluding OTUs with $<100$ total sequences). Most orchid mycorrhizal sequences were related to members of Mycena (one OUT - 164772 sequences - $95.5 \%$ ) in the protocorm samples, and Gymnopus (one OTU - 90028 sequences - $53.3 \%$ ) in the adult root samples. It is noteworthy that fungal partners differed between protocorm and adult stages in $G$. confusoides, in which a single Mycena (OTU3) was dominant and occurred exclusively in protocorms, and one prevailing Gymnopus (OTU5) that occurred solely in roots (Table S2). Raw sequences reads were deposited in NCBI SRA database under BioProject xxxxx and representative sequences for each putative OMFs OUT found in this study were submitted to GenBank nucleotide database under the Accession Numbers (xxxxx).

qPCR standard curves based on known concentrations of target DNA showed a linear correlation (OTU3, $r^{2}=0.998$; OTU $\left.5, r^{2}=0.997\right)$ between $\log$ values of input DNA and qPCR threshold cycles over at least five orders of magnitude (Fig. S2B, D), enabling accurate quantification of our target fungi in terms of target DNA molecules (mol) per $\mu \mathrm{L}$ DNA extract (Table S3). The highest concentrations of target OrM DNA were obtained from $G$. confusoides protocorm and root samples, with the average OTU3 concentration being $640129.5 \mathrm{~mol} \mu 1^{-1}$ DNA extract $\left(\mathrm{SE}=43787.6 \mathrm{~mol} \mu 1^{-1}\right)$ and the average OTU5 concentration being $484626.5 \mathrm{~mol} \mu 1^{-1}$ DNA extract $\left(\mathrm{SE}=62923 \mathrm{~mol} \mu 1^{-1}\right.$ ), respectively. For these two target OTUs, no significant relationship between the distance from the orchid plant and fungal concentration was observed in bamboo litter surrounding the orchid plant $\left(\chi^{2}=4.40\right.$ and $6.39, p>0.05$ for OTU3 and OTU5, respectively) (Fig. S3). In both cases, plot explained a minor part $(<10 \%)$ of the total variation, indicating that the results were consistent among plots.

\subsection{Germination}

Seed germination was regularly encountered at the study site and distinct stages of seedling development (protocorms and young root tubers) were observed (Fig. 2A,B). The percentage of packets containing germinating seeds varied between $70 \%$ in plot 1, $90 \%$ in plot 2, $85 \%$ in plot 3 and $65 \%$ in plot 4 . On

average, 3.2 protocorms ( $0.64 \%$ seed germination) were found within individual seed packets. Analysis of data for germination of seed packets showed no significant effect $\left(\chi^{2}=4.58 ; p=0.33\right)$ of distance to adult 
plants within the $10-\mathrm{m}^{2}$ plots (Fig. 3), nor was there any relationship between the four directions in which seed packages were buried in the litter (data not shown). A strong, significant relationship $\left(\chi^{2}=51.91 ; p<\right.$ 0.001) was observed between seed germination and abundance of Mycena in the litter, but not between seed germination and abundance of Gymnopus $\left(\chi^{2}=0.04, p=0.84\right)$ (Fig. 4).

\section{DISCUSSION}

\subsection{Mycorrhizal communities associating with Gastrodia confusoides}

Autotrophic and mycoheterotrophic orchids tend to associate with different sets of mycorrhizal fungi (Jacquemyn \& Merckx, 2019; Wang et al., 2021). Whereas most autotrophic orchids typically associate with a wide variety of fungi including so-called rhizoctonia fungi (i.e. members of Tulasnellaceae, Ceratobasidiaceae and Serendipitaceae), partially and fully mycoheterotrophic orchids associate predominantly with ectomycorrhizal fungi (Ogura-Tsujita et al., 2012; Těšitelová et al., 2015; Yagame et al., 2016) and saprotrophic fungi (Lee et al., 2015; Ogura-Tsujita et al., 2018). Our results are in line with these observations and showed that the dominant fungi associating with $G$. confusoides were saprotrophic fungi of the Mycenaceae and Marasmiaceae (Table S2).

These results further confirm previous reports that have shown that fungi from Mycenaceae and Marasmiaceae are the two major fungal families associating with Gastrodia species: Gastrodia confusa-Mycena of Mycenaceae (Ogura-Tsujita et al., 2009), Gastrodia sesamoides - Marasmius and Campanella of Marasmiaceae (Dearnaley \& Bougoure, 2010), Gastrodia appendiculata - Mycena of Mycenaceae (Lee et al., 2015), Gastrodia fontinalis - Gymnopus of Marasmiaceae (Lee et al., 2015) and Gastrodia nantoensis - Mycena of Mycenaceae (Lee et al., 2015). In Japan, members of Marasmiaceae and Mycenaceae were also the dominant fungal partners associating with threeGastrodia species (G. confusa, Gastrodia pubilabiata, and Gastrodia nipponica) growing in different vegetation types (Kinoshita et al., 2016). However, individuals of G. nipponica inhabiting broadleaved forests were shown to simultaneously associate with ectomycorrhizal fungi from Russulaceae and Sebacinaceae, suggesting that orchid mycorrhizal communities can vary depending on the vegetation type where the species grow. A similar result was found in Gastrodia elata, which is mainly found in broadleaved forests. Chen et al. (2019) showed that besides Mycenaceae, a broader spectrum of fungal associations, such as Resinicium, Hyphodontia, Sistotrema, Tricholoma andRussula was detected in the juvenile stage of G. elata .

The Mycena (OTU3) sequences isolated from G. confusoidesprotocorms shared high sequence similarity with sequences isolated from $G$. nantoensis and $G$. appendiculata growing in other bamboo forest (Lee et al., 2015). These results suggest that this Mycenastrain is particularly adapted to grow in bamboo forest, where it decomposes bamboo litter, and therefore could be shared amongGastrodia species. It has been reported that only a limited group of saprotrophic fungi can occur in bamboo forests in Japan, and the fungal community in bamboo forests is different from that in other forest types (Shidei, 1974; Yashima \& Nose, 2010). These results suggest the adaptation of "the bamboo forest Gastrodia species" to particular fungal groups specific to bamboo thickets.

\subsection{The shift of fungal partner in different developmental stages}

Although it is clear that mycorrhizal interactions play a crucial role in the life cycle of orchids (Rasmussen, 1995), there is remarkable variation in the number of fungi orchids associate with, ranging from highly specific interactions with one or a few OrM fungi (McCormick et al., 2009) to very broad interactions with orchids associating with multiple fungi simultaneously (e.g. Jacquemyn et al., 2012c; De Long et al., 2013; Waud et al., 2017; Shefferson et al., 2019). Furthermore, there is growing evidence that orchid mycorrhizal interactions are not stable, but can vary substantially both in space (Xing et al., 2020) and time (Bidartondo \& Read, 2008), with some species continuously associating with the same partner while others show almost complete switching of partners through space or time (Ventre-Lespiaucq et al., 2021). Spatial and temporal turnover may reflect variation in ecological conditions or fungal availability. Temporal turnover can occur throughout the growing season or between subsequent years (e.g. McCormick et al., 2006; Ercole et al., 2015; Oja et al., 2015), or between ontogenetic stages (e.g. McCormick et al., 2004; Bidartondo \& Read, 
2008; Jacquemyn et al., 2011). For example, Tipularia discolor adults associate with a variety of fungi, while fungal diversity detected in protocorms was much lower (McCormick et al., 2004). A recent meta-analysis has shown that temporal turnover of mycorrhizal partners occurs frequently in orchids and that in most cases partial replacement, whereby an individual retains a subset of its fungal partners and replaces others, was the most frequent scenario (Ventre-Lespiaucq et al., 2021).

Our high-throughput sequencing data demonstrated that $G$. confusoides switched fungal partners from the protocorm stage (Mycenaceae) to the adult stage (Marasmiaceae). A similar shift in fungal partner across ontogenetic stages has been observed in G. elata using an in vitro culture system, in which Mycenapromoted seed germination and the further development of the tuber required the association with Armillaria (Xu \& Guo, 2000). However, high-throughput sequencing of both juvenile and adult stages of G. elata showed that the juvenile stage associated with more diverse fungal groups than Mycena only (Chen et al., 2019). A similar pattern of total replacement has been found in Cyrtosia septentrionalis, another mycoheterotrophic orchid associated with wood-decaying fungi. In this case, Physisporinus induces seed germination, while the adult stage associates with Armillaria(Umata et al., 2013).

Complete partner turnover seems to occur primarily in subtropical Asian mycoheterotrophic orchids associated with litter- or wood-decaying fungi, but not in temperate mycoheterotrophic orchids. In temperate forests, mycoheterotrophic orchids, such as Cephalanthera austiniae, Corallorhiza species and Neottia nidus-avisusually associate with narrow clades of ectomycorrhizal fungi during their entire life cycle (Taylor \& Bruns, 1997; McKendrick et al., 2000, 2002). Although a complete shift in fungal partners across different life stages may come with severe costs (Ventre-Lespiaucq et al., 2021) and may pose severe constraints on the population dynamics of mycoheterotrophic orchids, switching partners can be expected to occur when it provides serious benefits in terms of resource acquisition and allocation (Bever, 2015). In Pinus sylvestris, shifts in ectomycorrhizal fungal community composition provide individual trees the abiliity to access more diverse nutrient pools for sustaining plant productivity (Kyaschenko et al., 2017). For the rapid growth of G. elata tuber, changing fungal partners to Armillaria may enable the utilization of a larger carbon pool, i.e. the wood in the forest (Ogura-Tsujita et al., 2021). Similar differences in resource acquisition abilities may explain the observed switch in mycorrhizal partners in protocorms and adult plants of $G$.confusoides .

\subsection{Abundance and spatial distribution of mycorrhizal fungi and seed germination}

The distribution and abundance of suitable OrM fungi in the soil are critical factors determining seed germination and hence the spatial distribution of orchids within sites (McCormick \& Jacquemyn, 2014). Whereas a number of studies have shown strong relationships between the presence/absence of adult plants and seed germination (McKendrick et al., 2000, 2002; Batty et al., 2001; Diez, 2007; Jacquemyn et al., 2007, 2012a,b), we found no significant relationship between the distance from the mother plant and germination (Fig. 3), indicating that locations closer to adult plants are not necessarily locations supporting higher abundances of mycorrhizal fungi required for seed germination. This can be explained by the fact that adults plants almost exclusively associate with Gymnopus, which was completely absent in germinating seeds and protocorms. We also found no correlation between seed germination and the abundance of Gymnopus (OTU5) (Fig. 4), suggesting that this fungus is not involved in seed germination, but only starts to play a role in the subsequent growth of seedlings into adult plants. In contrast, protocorms associated mainly with Mycena (OTU3) and in this case there was a highly significant, positive relationship between seed germination and the abundance of Mycena (Fig. 4), indicating that the local distribution of this fungus is the determining factor driving spatial patterns of seed germination.

These differences in seed germination patterns between autotrophic and mycoheterotrophic orchids may be explained by the nutritional needs of different groups of OrM fungi they associate with. In orchids associating with rhizoctonia fungi, the accumulation of fungi around adults plants and the associated strong relationship between germination and the location of adult plants (Waud et al., 2016) can be explained by the fact that the orchids contribute carbon to and hence support the fungi (Cameron et al., 2008; Liebel et al., 2015). In orchids associating with ectomycorrhizal fungi, the fungi are supported by trees, and hence the distribution and abundance of the orchids is for a large part related to the distribution and abundance of Tomentella fungi 
and their ectomycorrhizal trees (McCormick et al., 2009). In bamboo forests, the ground is usually covered with a thick layer of bamboo litter. Since Mycena and Gymnopusare efficient decomposers of bamboo leaves, sticks and trunks for nutrient recycling, they are expected to have a random distribution within the bamboo forest.

\section{CONCLUSION}

Seed germination and subsequent growth to an adult plant in the mycoheterotrophic orchid G. confusoides represents a two-stage process that is accompanied by a prompt switch in mycorrhizal partners. Our results provide the first evidence that the spatial distribution and abundance of litter-decaying fungi are distributed randomly within the bamboo forest and independently from $G$. confusoides adults. Future research should investigate in more detail the functional and physiological basis of the interaction with both fungi and assess the cost of switching. In particular, more details about resource transport patterns between symbionts are needed to understand why adult plants switch to a different partner.

\section{ACKNOWLEDGEMENTS}

We thank Dr. C. K. Yang for providing the photograph of blooming Gastrodia confusoides plants.

\section{DATA ACCESSIBILITY}

\section{AUTHOR CONTRIBUTIONS}

Y.I.L. designed the research. Y.Y.L. and Y.H.C. contributed to molecular experiments. Y.I.L., Y.Y.L. and M.B. performed bioinformatic analyses. H.J. conducted statistic analyses. Y.I.L. and H.J. wrote the manuscript.

\section{REFERENCES}

Adams, R. I., Miletto, M., Taylor, J. W., et al. (2013). Dispersal in microbes: fungi in indoor air are dominated by outdoor air and show dispersal limitation at short distances. ISME Journal, 7,1262-1273.

Altschul, S. F., Gish, W., Miller, W., Myers, E. W., \& Lipman, D. J. (1990). Basic local alignment search tool. Journal of Molecular Biology, 215, 403-410.

Arditti, J., \& Ghani, A. K. A. (2000). Tansley Review No. 110. Numerical and physical properties of orchid seeds and their biological implications. New Phytologist, 145, 367-421.

Batty, A. L., Dixon, K. W., Brundrett, M., \& Sivasithamparam, K. (2001). Constraints to symbiotic germination of terrestrial orchid seed in a Mediterranean bushland. New Phytologist, 152, 511-520.

Benson, D. A., Karsch-Mizrachi, I., Lipman, D. J., Ostell, J., \& Sayers, E. W. (2009). GenBank. Nucleic Acids Research, 37 (Database issue), D26-31.

Bever, J. D. (2015). Preferential allocation, physio-evolutionary feedbacks, and the stability and environmental patterns of mutualism between plants and their root symbionts. New Phytologist, 205, 1503-1514.

Bidartondo, M. I., \& Read, D. J. (2008). Fungal specificity bottlenecks during orchid germination and development. Molecular Ecology, 17,3707-3716.

Cameron, D. D., Johnson, I., Read, D. J., \& Leake, J. R. (2008). Giving and receiving: measuring the carbon cost of mycorrhizas in the green orchid, Goodyera repens . New Phytologist, 180, 176-184.

Chen, L., Wang, Y. C., Qin, L. Y. et al. (2019). Dynamics of fungal communities during Gastrodia elata growth. BMC Microbiology 19, 158.

Clark, C. J., Poulsen, J. R., Levey, D. J., \& Osenberg, C. W. (2007). Are plant populations seed limited? A critique and meta-analysis of seed addition experiments. American Naturalist, 170, 128-142.

Condit, R., Hubbell, S. P., \& Foster, R. B. (1992). Recruitment near conspecific adults and the maintenance of tree and shrub diversity in a neotropical forest. American Naturalist, 140, 261-286. 
Dearnaley, J. D. W., \& Bougoure, J. J. (2010). Isotopic and molecular evidence for saprotrophic Marasmiaceae mycobionts in rhizomes of Gastrodia sesamoides . Fungal Ecology, 3, 288-294.

De Long, J. R., Swarts, N. D., Dixon, K. W., \& Egerton-Warburton, L. M. (2013). Mycorrhizal preference promotes habitat invasion by a native Australian orchid: Microtis media. Annals of Botany, 111,409-418.

Diez, J. M. (2007). Hierarchical patterns of symbiotic orchid germination linked to adult proximity and environmental gradients. Journal of Ecology, 95, 159-170.

Edgar, R. C. (2013). UPARSE: highly accurate OTU sequences from microbial amplicon reads. Nature Methods, 10, 996.

Ercole, E., Adamo, M., Rodda, M., Gebauer, G., Girlanda, M., \& Perotto S. (2015). Temporal variation in mycorrhizal diversity and carbon and nitrogen stable isotope abundance in the wintergreen meadow orchidAnacamptis morio . New Phytologist , 205, 1308-1319.

Eriksson, O., \& Ehrlen, J. (1992). Seed and microsite limitation of recruitment in plant populations. Oecologia, 91, 360-364.

Getzin, S., Wiegand, T., \& Hubbell, S. P. (2014). Stochastically driven adult-recruit associations of tree species on Barro Colorado Island.Proceedings of the Royal Society of London B: Biological Sciences, 281, 20140922 .

Hsu, T. C. (2008). Taxonomy of Gastrodia (Orchidaceae) in Taiwan. Master thesis, National Taiwan University, Taipei.

Jacquemyn, H., Brys, R., Vandepitte, K., Honnay, O., Roldan-Ruiz, I., \& Wiegand, T. (2007). A spatially explicit analysis of seedling recruitment in the terrestrial orchid Orchis purpurea . New Phytologist, 176, 448-459.

Jacquemyn, H., Merckx, V., Brys, R., Tyteca, D., Cammue, B. P. A., Honnay, O., \& Lievens, B. (2011). Analysis of network architecture reveals phylogenetic constraints on mycorrhizal specificity in the genusOrchis (Orchidaceae). New Phytologist, 192, 518-528.

Jacquemyn, H., Brys, R., Honnay, O., Roldan-Ruiz, I., Lievens, B., \& Wiegand, T. (2012a). Non-random spatial structuring of orchids in a hybrid zone of three Orchis species. New Phytologist, 193,454-464.

Jacquemyn, H., Brys, R., Lievens, B., \& Wiegand, T. (2012b). Spatial variation in below ground seed germination and divergent mycorrhizal associations correlate with spatial segregation of three co-occurring orchid species. Journal of Ecology, 100, 1328-1337.

Jacquemyn, H., Deja, A., Dehert, K., Cachapa Bailarote, B.,\& Lievens, B. (2012c). Variation in mycorrhizal associations with Tulasnelloid fungi among populations of five Dactylorhiza species. PLoS ONE, 7, e42212.

Jacquemyn, H., \& Merckx, V. S. F. T. (2019). Mycorrhizal symbioses and the evolution of trophic modes in plants. Journal of Ecology,107, 1567-1581.

Johansson, V. A. \& Ove Eriksson, O. (2013). Recruitment limitation, germination of dust seeds, and early development of underground seedlings in six Pyroleae species. Botany, 91, 17-24.

Kinoshita, A., Ogura-Tsujita, Y., Umata, H., Sato, H., Hashimoto, T., \& Yukawa, T. (2016). How do fungal partners affect the evolution and habitat preferences of mycoheterotrophic plants? A case study in Gastrodia . American Journal of Botany, 103, 207-220.

Kyaschenko, J., Clemmensen, K., Hagenbo, A. et al. (2017). Shift in fungal communities and associated enzyme activities along an age gradient of managed Pinus sylvestris stands. ISME Journal, 11, 863-874.

Kusano, S. (1911). Gastrodia elata and its symbiotic association with Armilllaria mellea. Journal of College of Agriculture Imperial University of Tokyo, 4, 1-66. 
Lee, Y. I., Yang, C. K., \& Gebauer, G. (2015). The importance of associations with saprotrophic nonRhizoctonia fungi among fully mycoheterotrophic orchids is currently under-estimated: novel evidence from sub-tropical Asia. Annals of Botany, 116, 423-435.

Liebel, H. T., Bidartondo, M. I., \& Gebauer, G. (2015). Are carbon and nitrogen exchange between fungi and the orchid Goodyera repensaffected by irradiance? Annals of Botany, 115, 251-261.

Martos, F., Dulormne, M., Pailler, T., Bonfante, P., Faccio, A., Fournel, J., \& Selosse, M. A. (2009). Independent recruitment of saprotrophic fungi as mycorrhizal partners by tropical achlorophyllous orchids. New Phytologist, 184, 668-681.

McCormick, M. K., Whigham, D. F. \& O’Neill, J. P. (2004). Mycorrhizal diversity in photosynthetic terrestrial orchids. New Phytologist, 163, 425-438.

McCormick, M. K., Whigham, D. F., Sloan, D., O'Malley, K. \& Hodkinson, B. (2006). Orchid-fungus fidelity: a marriage meant to last?Ecology, 87, 903-911.

McCormick, M. K., Whigham, D. F., O'Neill, J. P. et al. (2009). Abundance of Corallorhiza odontorhiza (Orchidaceae) within an established population reflects abundance of ectomycorrhizal root tips and fungal community composition. Ecological Monographs, 79,619-635.

McCormick, M. K., Taylor, D. L., Juhaszova, K., Burnett, R. K., Whigham, D. F., \& O'Neill, J. (2012). Limitations on orchid recruitment: not a simple picture. Molecular Ecology, 21, 1511-1523.

McCormick, M. K., \& Jacquemyn, H. (2014). What constrains the distribution of orchid populations? New Phytologist, 202,392-400.

McCormick, M. K., Taylor, D. L., Whigham, D. F., \& Burnett, R. K. Jr. (2016). Germination patterns in three terrestrial orchids relate to abundance of mycorrhizal fungi. Journal of Ecology, 104, 744-754.

McCormick, M. K., Whigham, D. F., \& Canchani-Viruet, A. (2018). Mycorrhizal fungi affect orchid distribution and population dynamics. New Phytologist, 219, 1207-1215.

McKendrick, S. L., Leake, J. R., Taylor, D. L., \& Read, D. J. (2000). Symbiotic germination and development of myco-heterotrophic plants in nature: ontogeny of Corallorhiza trifida and characterization of its mycorrhizal fungi. New Phytologist, 145, 523-537.

McKendrick, S. L., Leake, J. R., Taylor, D. L., \& Read, D. J. (2002). Symbiotic germination and development of the mycoheterotrophic orchidNeottia nidus-avis in nature and its requirement for locally distributed Sebacina spp. New Phytologist, 154, 233-247.

Munzbergova, Z., \& Herben, T. (2005). Seed, dispersal, microsite, habitat and recruitment limitation: identification of terms and concepts in studies of limitations. Oecologia, 145, 1-8.

Nathan, R., \& Muller-Landau, H. C. (2000). Spatial patterns of seed dispersal, their determinants and consequences for recruitment. Trends in Ecology \& Evolution, 15, 278-285.

Ogura-Tsujita, Y., Gebauer, G., Hashimoto, T., Umata, H., \& Yukawa, T. (2009). Evidence for novel and specialized mycorrhizal parasitism: the orchid Gastrodia confusa gains carbon from saprotrophicMycena . Proceedings of the Royal Society of London Series B-Biological Sciences, 276, 761-767.

Ogura-Tsujita, Y., Gebauer, G., Xu, H., Fukasawa, Y., Umata, H., Tetsuka, K., Kubota, M., Schweiger, J. M. I., Yamashita, S., Maekawa, N., Maki, M., Isshiki, S., \& Yukawa, T. (2018).The giant mycoheterotrophic orchid Erythrorchis altissima is associated mainly with a divergent set of wood-decaying fungi. Molecular Ecology, 27, 1324-1337.

Ogura-Tsujita, Y., Yukawa, T., \& Kinoshita, A. (2021). Evolutionary histories and mycorrhizal associations of mycoheterotrophic plants dependent on saprotrophic fungi. Journal of Plant Research, 134,19-41. 
Oja, J., Kohout, P., Tedersoo, L., Kull, T., \& Koljalg U. (2015). Temporal patterns of orchid mycorrhizal fungi in meadows and forests as revealed by 454 pyrosequencing. New Phytologist , 205, 1608-1618.

Poulsen, J. R., Osenberg, C. W., Clark, C. J., Levey, D. J., \& Bolker, B. M. (2007). Plants as reef fish: fitting the functional form of seedling recruitment. American Naturalist, 170, 167-183.

Pridgeon, A. M., Cribb, P. J., Chase, M. W., \& Rasmussen, F. N. (2005). Genera Orchidacearum, vol. 4: Epidendroideae (part 1). Oxford University Press, Oxford, UK.

Rasmussen, H. N. (1995). Terrestrial orchids: from seed to mycotrophic plant. Cambridge University Press, New York, NY, USA.

Rasmussen, H. N., \& Whigham, D. F. (1993). Seed ecology of dust seeds in situ: a new study technique and its application in terrestrial ecology. American Journal of Botany, 80, 1374-1378.

Rasmussen, H. N., \& Whigham, D. F. (1998). The underground phase: a special challenge in studies of terrestrial orchid populations.Botanical Journal of the Linnean Society, 126, 49-64.

Rasmussen, H. N., \& Rasmussen, F. N. (2009). Orchid mycorrhiza: implications of a mycophagous life style. Oikos, 118, 334-345.

Rasmussen, H. N., Dixon, K. W., Jersakova, J., \& Těšitelová, T. (2015). Germination and seedling establishment in orchids: a complex of requirements. Annals of Botany, 116, 391-402.

Shefferson, R. P., Bunch, W., Cowden, C. C., Lee, Y. I., Kartzinel, T. R., Yukawa, T., Downing, J., \& Jiang, H. (2019). Does evolutionary history determine specificity in broad ecological interactions? Journal of Ecology, 107, 1582-1593.

Shidei, T. (1974). Report of fungal flora in bamboo forest. Transactions of Mycological Society of Japan, 15, 251-257 (in Japanese).

Smith, S. E., \& Read, D. J. (2008). Mycorrhizal symbiosis. 3rd ed. New York, NY, \& London, UK: Academic Press.

Taylor, D. L., \& Bruns, T. D. (1997). Independent, specialized invasions of ectomycorrhizal mutualism by two nonphotosynthetic orchids.Proceedings of the National Academy of Sciences, USA, 94, 4510-4515.

Tesitelova, T., Kotilinek, M., Jersakova, J. et al. (2015). Two widespread green Neottia species (Orchidaceae) show mycorrhizal preference for Sebacinales in various habitats and ontogenetic stages.Molecular Ecology, 24, 1122-1134.

Umata, H., Ota, Y., Yamada, M. et al (2013). Germination of the fully myco-heterotrophic orchid Cyrtosia septentrionalis is characterized by low fungal specificity and does not require direct seed-mycobiont contact. Mycoscience, 54, 343-352.

Ventre-Lespiaucq, A., Jacquemyn, H., Rasmussen, H. N., \& Méndez, M. (2021). Temporal turnover in mycorrhizal interactions: a proof of concept with orchids. New Phytologist, 230, 1690-1699..

Wang, B. C., \& Smith, T. B. (2002). Closing the seed dispersal loop.Trends in Ecology E Evolution, 17, 379-386.

Wang, D., Jacquemyn, H., Gomes, S. I. F., Vos, R. A., \& Merckx, V. S. F. T. (2021). Symbiont switching and trophic mode shifts in Orchidaceae.New Phytologist, in press. https://doi.org/10.1111/nph.17414.

Waud, M., Wiegand, T., Brys, R., Lievens, B., \& Jacquemyn, H. (2016). Nonrandom seedling establishment corresponds with distance-dependent decline in mycorrhizal abundance in two terrestrial orchids. New Phytologist, 211, 255-264.

Waud, M., Brys, R., Van Landuyt, W., Lievens, B., \& Jacquemyn, H. (2017). Mycorrhizal specificity does not limit the distribution of an endangered orchid species. Molecular Ecology, 26, 1687-1701. 
WCSP. (2014). World checklist of selected plant families. Facilitated by the Royal Botanic Gardens, Kew. Published on the Internet: http://apps.kew.org/wcsp/.

Xing, X., Liu, Q., Gao, Y., Shao, S., Guo, L., Jacquemyn, H., Zhao, Z., \& Guo, S. (2020). The architecture of the network of orchid-fungus interactions in nine co-occurring Dendrobium species.Frontiers in Ecology and Evolution, 8, 130.

$\mathrm{Xu}$, J., \& Guo, S. (2000). Retrospect on the research of the cultivation of Gastrodia elata Bl, a rare traditional Chinese medicine. Chinese Medical Journal, 113, 686-692.

Yagame, T., Ogura-Tsujita, Y., Kinoshita, A. et al. (2016). Fungal partner shifts during the evolution of mycoheterotrophy inNeottia . American Journal of Botany, 103, 1630-1641.

Yashima, T., \& Nose, I. (2010). Changes of macrofungal flora along the degree of mixture of Moso-bamboo thicket and broad-leaved forest.Annual Report of the Ishikawa Prefectural Forest Research, 42,34-37. (in Japanese).

\section{Supporting/Supplemental information}

Table S1 List of primers used in this study.

Table S2 Summary of fungal operational taxonomic units (OTUs) detected in orchid protocorms and roots using the Illumina Miseq platform.

Table S3 Mean concentration of OTU3 (Mycena) and OTU5 (Gymnopus ) DNA (mol $\mu \mathrm{l}^{-1}$ ) including standard deviation of the mean quantified using qPCR analysis of Gastrodia confusoides protocorms, roots and surrounding bamboo litter samples obtained in four cardinal directions and at five radial distances (5, $50,100,200$ and $500 \mathrm{~cm}$ ).

Fig. S1 Radial sampling plan applied for the seed germination experiment and the collection of bamboo litter surrounding Gastrodia confusoides individuals.

Fig. S2 Quantitative PCR results for OTU3 (Mycena) and OTU5 (Gymnopus) detected in Gastrodia confusoidesprotocorms or roots, including melting curve and standard curve analyses.

Fig. S3 For Mycena (OTU3) and Gymnopus (OTU5), no significant relationship between the distance from the orchid plant and fungal abundance determined by $\mathrm{qPCR}$ was observed in bamboo litter surrounding the orchid plant $\left(\chi^{2}=4.40\right.$ and $6.39, p>0.05$ for OTU3 and OTU5, respectively).

\section{List of Figures}

Fig. 1. Gastrodia confusoides in its natural habitat. (A) Adult plants with blooming flowers growing between bamboo litter. Scale bar $=1 \mathrm{~cm}$. (B) Stem elongation after flowers have been successfully pollinated. Scale bar $=10 \mathrm{~cm}$. (C) After two weeks of pollination, mature fruits have opened and released seeds (arrows). Scale bar $=1 \mathrm{~cm}$. (D) The tube with mycorrhizal roots (arrows) in decaying bamboo litter. Scale bar $=$ $1 \mathrm{~cm}$. (E) Light microscopy section showing the presence of mycorrhizal fungi in cortical cells of roots. E, epidermal cells; C, cortical cells; H, fungal hyphae. Scale bar $=10 \mu \mathrm{m}$.

Fig. 2. Different stages in the germination process of Gastrodia confusoides seeds and indications of the presence of mycorrhizal fungi. (A) Developing protocorms from a single packet at 12 months after sowing. Scale bar $=2 \mathrm{~mm}$. (B) A selection of different stages of protocorms and young root tubers from the packet. Scale bar $=2 \mathrm{~mm}$. (C) At the early stage of germination, fungal hyphae (arrow) have colonized the basal part of embryo. Scale bar $=50 \mu \mathrm{m}$ (D) The enlarged embryo has ruptured the seed coat. Scale bar $=50$ $\mu \mathrm{m}(\mathrm{E})$ The developing protocorm has elongated with the meristematic cells at the up region and the fungal colonization at the basal region. Fungal hyphae (arrows). Scale bar $=50 \mu \mathrm{m}$.

Fig. 3. Relationship between the percentage seed germination of Gastrodia confusoides and distance from adult plants $(\mathrm{cm})$ measured in four plots in bamboo forest in Taiwan. 
Fig. 4. Relationship between the abundance of Mycena andGymnopus $\left(\mathrm{mol} \mu \mathrm{L}^{-1}\right)$ and percentage seed germination of Gastrodia confusoides. Seed germination was expressed as the percentage of seeds developing into a protocorm.

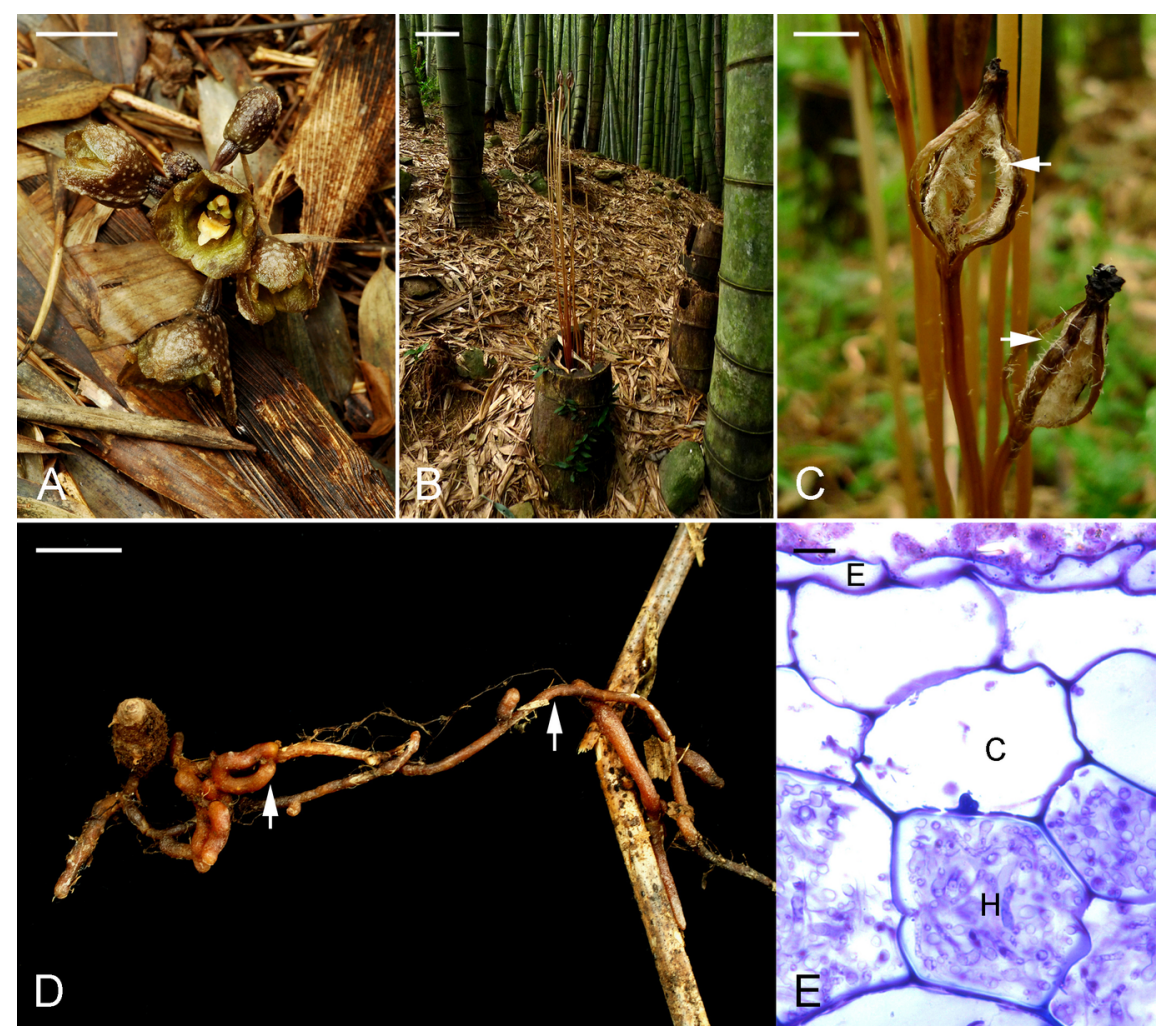




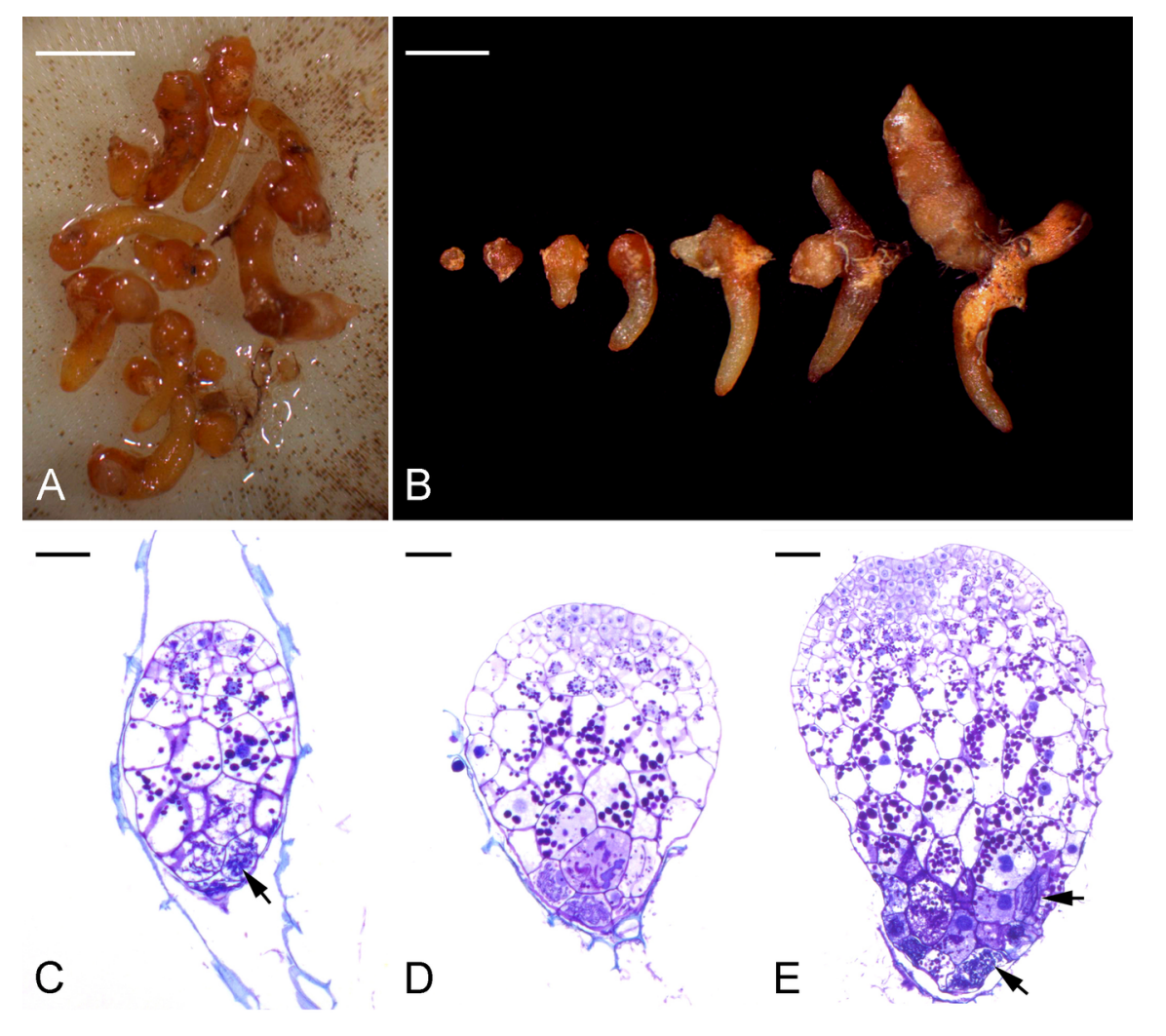




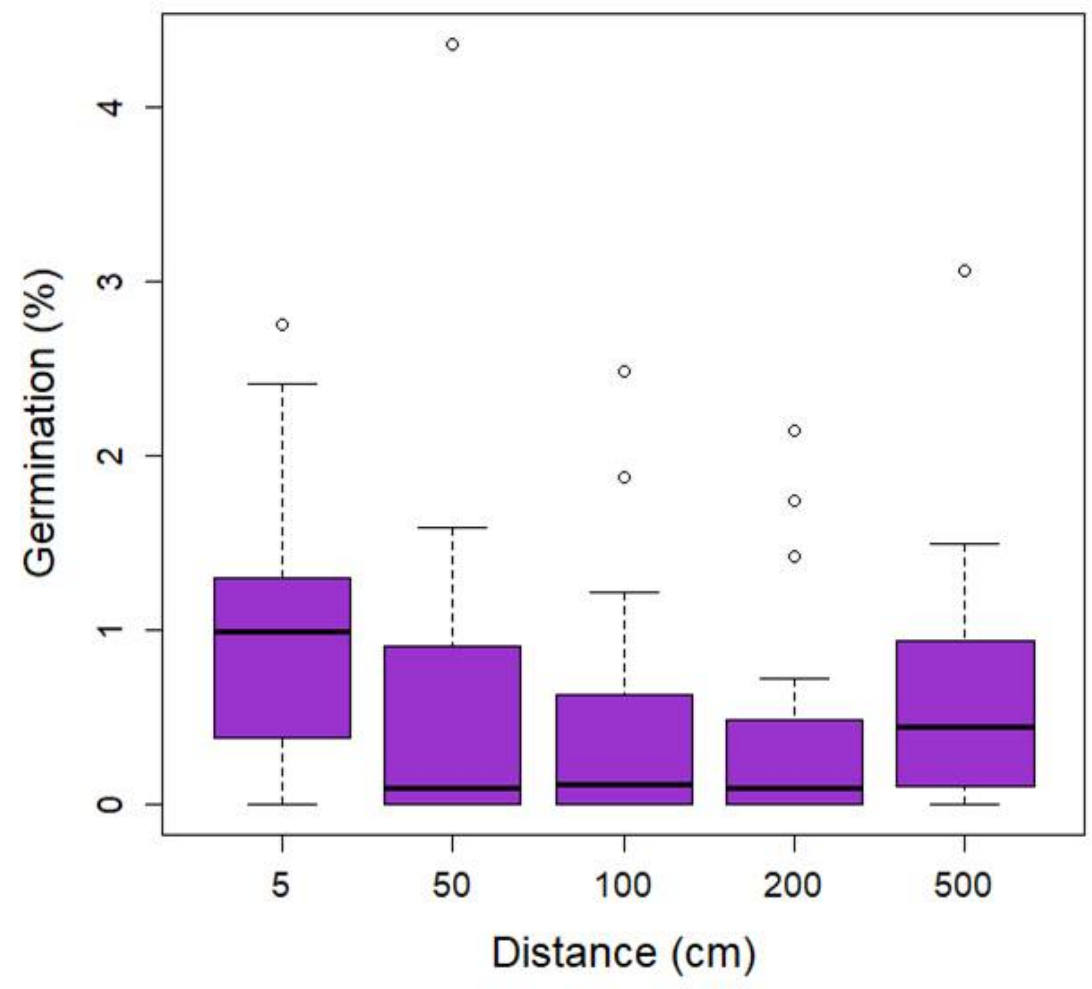



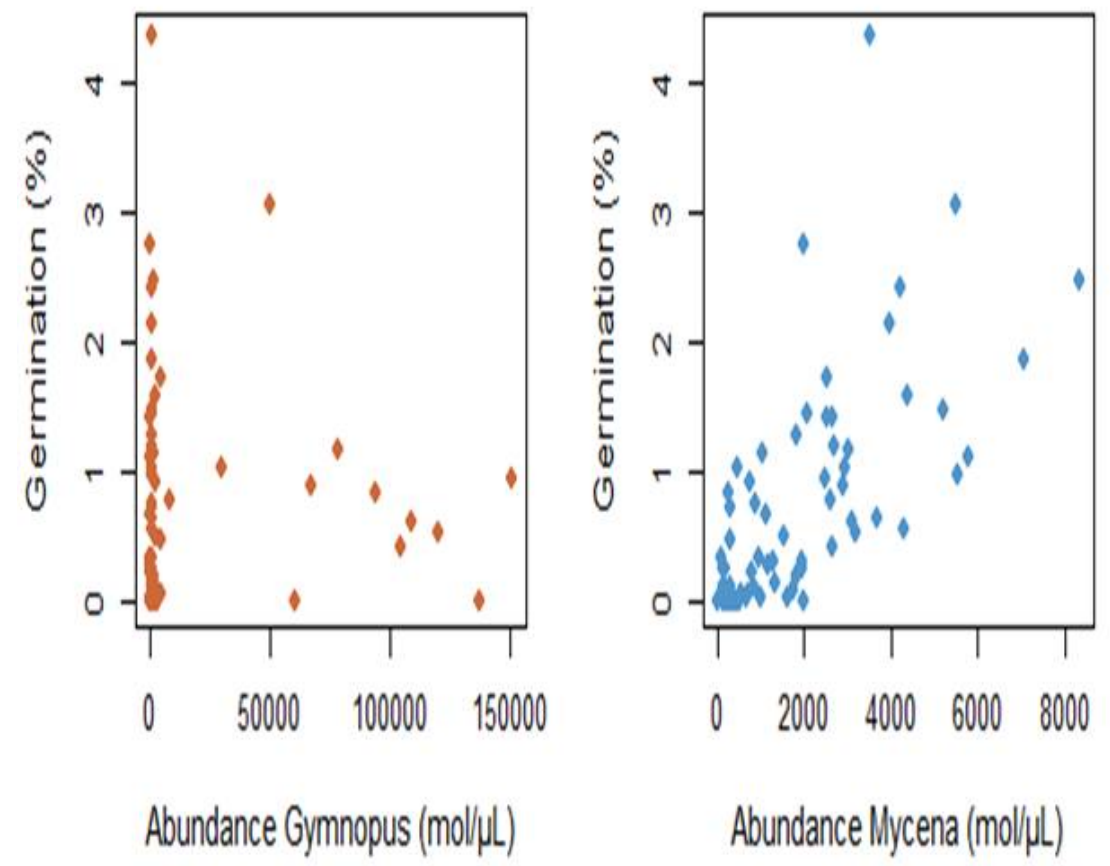

Abundance Gymnopus (mol/pLL) Abundance Mycena (mol/ul) 